\title{
CARACTERIZACIÓN PARAMÉTRICA DE LOS RENDIMIENTOS DE LOS PRECIOS DEL PETRÓLEO 2010-2015
}

\author{
Parametric Characterization \\ YIELDS PRICES OIL 2010-2015 \\ Martha Beatriz Mota-Aragón* \\ Leovardo Mata-Mata*
}

(Recibido: septiembre, 2015/Aceptado: diciembre, 2015)

\section{Resumen}

En este trabajo se caracterizan los rendimientos de los precios del petróleo Brent, West Texas Intermediate y la mezcla mexicana de exportación mediante los parámetros de una distribución de probabilidad no normal. Se revisa el periodo 2010-2015 en dos ventanas de tiempo: precios de crudo bajos y precios de crudo altos. Se encuentra evidencia de un ajuste adecuado de la distribución hiperbólica generalizada para ambos subperiodos y, por, ende se puede analizar el comportamiento poblacional de los rendimientos mediante cambios en los parámetros de la distribución de probabilidad.

Palabras clave: precios del petróleo, distribución hiperbólica generalizada, máxima verosimilitud, intervalo de confianza.

Clasificación JEL: C16, G11, G23.

* Profesora-investigadora de la Universidad Autónoma Metropolitana, Unidad Iztapalapa, Departamento de Economía. Correo electrónico: beatrizmota4@gmail.com. Correspondencia: Universidad Autónoma Metropolitana Avenida San Rafael Atlixco 186, col. Vicentina 09340, Del. Iztapalapa, México, Ciudad de México, Tel. 580447 75, Correo electrónico: beatrizmota4@gmail.com.

* Postdoctorante en la Universidad Autónoma Metropolitana, Unidad Iztapalapa, Departamento de Economía, Ciudad de México. Correo electrónico: leovardomata@gmail.com. 


\section{Abstract}

In this paper yields oil prices Brent, West Texas Intermediate and Mexican export mix are characterized by the parameters of a no-normal probability distribution. The period 2010-2015 is reviewed in two time windows: low oil prices and high crude prices. It is found evidence of an appropriate adjustment of the generalized hyperbolic distribution for both subperiods. Therefore can analyze the behavior of yields through changes in the parameters of the cumulative distribution.

Keywords: oil prices, generalized hyperbolic distribution, maximum likelihood, confidence interval.

JEL Classifications: C16, G11, G23.

\section{Introducción}

La historia económica mexicana muestra un antes y un después debido a la industria petrolera. La importancia que el petróleo tiene en la sociedad, cultura y economía del país es innegable. En este sentido, analizar el comportamiento del precio de la mezcla mexicana de petróleo de exportación es una tarea necesaria, pues brinda la posibilidad de que el sesgo procíclico del gasto se reduzca antes variaciones de la renta petrolera (IMP, 2011).

Las variaciones que experimenta la mezcla mexicana se relacionan con los cambios que experimentan otros tipos de petróleo crudo a nivel mundial (Cárdenas, 2009). Aunque si bien es cierto que en México existen tres variedades de petróleo crudo de exportación: itsmo, maya y olmeca. La mezcla de estos tres tipos de crudo constituye el principal petróleo de exportación (Brown, 1993), siendo predominante el petróleo maya en la composición de la mezcla, lo que dificulta y encarece la refinación (CEFP, 2009).

Por esta razón el precio de mercado de la mezcla mexicana (MME) tenderá a ser menor en comparación con los precios internacionales de las mezclas de otros países, como por ejemplo, el West Texas Intermediate (WTI) que suele usarse como precio de referencia en el mercado de Estados Unidos (SENER, 2012).

Alrededor del mundo se pueden encontrar cientos de tipos diferentes de petróleos, los cuales difieren por su calidad y dificultad para ser refinados. Sin embargo, en los mercados internacionales se encuentran algunos tipos 
de petróleo que resultan útiles al tratar de usarlos como referencia y que normalmente resultan ser los más importantes a nivel global.

Históricamente, la mezcla mexicana de petróleo de exportación ha sido comparada con el WTI, por ser el crudo de referencia en el mercado de los Estados Unidos y con el Brent, que es de gran importancia en los mercados europeos y uno de los más utilizados a nivel mundial (SENER, 2012).

Como se ha comentado en esta sección, los dos tipos de crudo más representativos a nivel mundial en los últimos años han sido el Brent y el WTI. Debido principalmente a la relevancia de países como Estados Unidos y el Reino Unido, quienes con sus respectivos crudos tratan de moldear el mercado y precio del petróleo a nivel global (CEFP, 2009).

En la figura 1 se muestra la estrecha relación que existe en el comportamiento y evolución entre los precios del WTI, Brent y MME en el mercado internacional de crudo. Esta relación es la que establece la pauta para analizarlos entre sí. Concretamente, se desea estudiar cómo cambia la distribución de probabilidad de cada tipo de petróleo al pasar del periodo de precios altos al periodo de precios bajos, que visualmente se puede apreciar en la gráfica es la transición entre el año 2013 y 2014.

Gráfica 1

Precios del petróleo Brent, WTI y MME 2010-2015

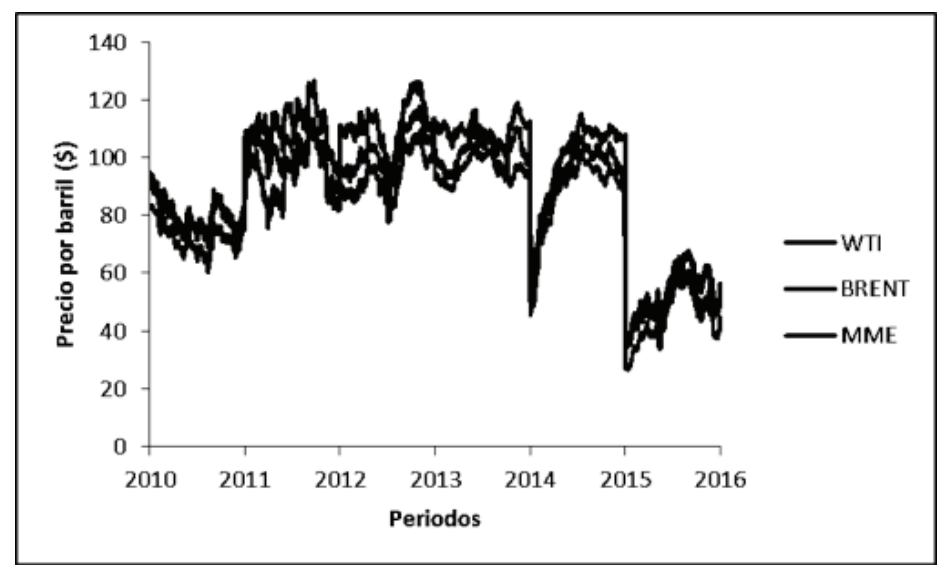

Fuente: elaboración propia con datos de Pemex.

En el siguiente apartado se describe la distribución de probabilidad propuesta para ajustar el comportamiento de los rendimientos de los precios del petróleo WTI, Brent y MME en el periodo 2010-2015. 
Este periodo se eligió de esta forma para no cargar con la volatilidad de la crisis financiera de 2008-2009, pues el objetivo solamente es analizar como cambian los parámetros de la distribución de probabilidad al pasar de una zona de precios altos a una zona de precios bajos. Adicionalmente, se presentan las pruebas de hipótesis para valorar la bondad de ajuste de la distribución de probabilidad para cada subperiodo de tiempo.

\section{Distribución de probabilidad y pruebas de hipótesis}

Una distribución de probabilidad acumulada versátil que puede capturar mediante sus parámetros diversos hechos estilizados de una serie de rendimientos es la distribución hiperbólica generalizada (DHG), (Protassov, 2004).

La DHG consta de diferentes bloques de construcción (Paolella, 2007). El primer componente es la función de Bessel de tercer orden con parámetro $\lambda$, la cual se define como:

$$
K_{\lambda}(x)=\frac{1}{2} \int_{0}^{\infty} t^{\lambda-1} e^{-\frac{1}{2} x\left(t+t^{-1}\right)} d t .
$$

donde $\lambda \in \mathbb{R}$ es un parámetro y el dominio de la función cumple $x>0$.

La función anterior permite definir a la variable aleatoria inversa gaussiana generalizada (IGG) como aquella que sigue la función de densidad, Barndorff-Nielsen (1977),

$$
\begin{aligned}
f(x ; \lambda, \chi, \psi)=\frac{1}{k_{\lambda}(\chi, \psi)} x^{\lambda-1} \exp \left[-\frac{1}{2}\left(\chi x^{-1}+\psi x\right)\right], \\
k_{\lambda}(\chi, \psi)=2 \eta^{\frac{\lambda}{2}} K_{\lambda}(\omega) \\
\eta=\chi / \psi \\
\omega=\sqrt{\chi \psi}
\end{aligned}
$$

donde los parámetros $\lambda \in \mathbb{R}, \chi \geq 0 \mathrm{y} \psi \geq 0$ determinan la localización y dispersión de las realizaciones $\lambda \in \mathbb{R}_{+}$de la variable aleatoria $X$.

Empleando los conceptos anteriores se define la función de densidad de una variable aleatoria hiperbólica generalizada como:

$$
f(x ; \lambda, \alpha, \beta, \delta, \mu)=\frac{k_{\lambda-1 / 2}\left((x-\mu)^{2}+\delta^{2}, \alpha^{2}\right)}{\sqrt{2 \pi} k_{\lambda}\left(\delta^{2}, \alpha^{2}-\beta^{2}\right)} \exp [\beta(x-\mu)],
$$


donde $\alpha, \delta \geq 0$ y $\lambda, \mu, \beta \in \mathbb{R}$ tales que $\beta \in[-\alpha, \alpha]$. La función de densidad IGG se encuentra implícita dentro de esta definición, y se vuelve explícita bajo las siguientes igualdades:

$$
\chi:=\delta^{2} \text {, }
$$

el valor esperado es: $\quad \psi:=\alpha^{2}-\beta^{2}$.

$$
E[X]=\mu+\beta \frac{k_{\lambda+1}(\chi, \psi)}{k_{\lambda}(\chi, \psi)},
$$

La varianza es: $\quad V[X]=\frac{k_{\lambda+1}(\chi, \psi)}{k_{\lambda}(\chi, \psi)}+\beta^{2} \frac{k_{\lambda}(\chi, \psi) k_{\lambda+2}(\chi, \psi)-\left[k_{\lambda+1}(\chi, \psi)\right]^{2}}{\left[k_{\lambda}(\chi, \psi)\right]^{2}}$.

Una breve explicación del significado de cada parámetro es como sigue:

a) $\alpha, \beta$ miden en gran medida el sesgo y la forma de la variable aleatoria. En particular, si $\beta$ es cero, entonces existe simetría en la DHG. El parámetro $\alpha$ moldea la curvatura alrededor de la moda de la variable aleatoria.

b) $\mu$ es un parámetro de localización, no es el valor esperado salvo que $\beta=0$.

c) $\delta$ mide el grado de curtosis de la DHG y moldea la forma de la distribución alrededor de la moda.

d) $\lambda$ es una medida de la forma de la DHG e influye en la dispersión que existe hacia ambas colas de la función de densidad.

En este documento se ajusta la DHG, pero antes se realiza una prueba para verificar que la variable aleatoria de interés sea estacionaria en el periodo de referencia. En particular, se realiza la prueba clásica de raíz unitaria DickeyFuller aumentada (Greene, 2012). Este paso es relevante, ya que garantiza la existencia de una distribución de probabilidad poblacional para las realizaciones de una variable aleatoria $X$. Esto se debe a que las series de tiempo cumplen en general con varianza finita, independiente de la ventana de tiempo, fluctúan alrededor de su media y presentan autocorrelaciones que disminuyen rápidamente con el número de rezagos (Hamilton, 1994).

La prueba de hipótesis de Dickey-Fuller define como afirmación base que existe raíz unitaria (no estacionariedad). De ahí que un modelo de regresión en series de tiempo sobre $y_{t}$ sea de la forma. 


$$
\Delta y_{t-1}=\mu+\gamma y_{t-1}+\delta_{1} \Delta y_{t-1}+\cdots+\delta_{p-1} \Delta y_{t-p+1}+\varepsilon_{t},
$$

pues de esa manera la prueba se basa en la significancia del coeficiente $\gamma$ (Tsay, 2010).

Una vez realizada la prueba de raíz unitaria se ajusta la función DHG bajo el método de máxima verosimilitud $(\mathrm{Hu}, 2005)$,

$$
\begin{aligned}
& L=\ln \left[f\left(x_{1}, x_{2}, \ldots, x_{n} ; \lambda, \alpha, \beta, \delta, \mu\right)\right] \\
& L=\sum_{i=1}^{n} \ln \left[f\left(x_{i} ; \lambda, \alpha, \beta, \delta, \mu\right)\right]
\end{aligned}
$$

donde se busca $\hat{\theta}=(\hat{\lambda}, Q, \hat{\beta}, \hat{\delta}, \rho)=\arg \operatorname{máx}\{L\}$.

Después se emplea la teoría asintótica clásica (Barndorff-Nielsen, 2012),

$$
\sqrt{n}(\hat{\theta}-\theta) \rightarrow N\left(0, I^{-1}\right)
$$

para calcular intervalos de confianza a cada uno de los parámetros estimados. La matriz de información $I$ se puede definir como $I=\operatorname{Cov}\left(S_{i}\right)$ siendo

$$
S_{i}=\frac{\partial \ln \left[f\left(x_{i} ; \theta\right)\right]}{\partial \theta}
$$

Por tanto, un intervalo de confianza de (1- $\alpha) \%$ para el parámetro estimado $\hat{v}$ se establece como

siendo

$$
\hat{v} \pm t_{\alpha / 2} \sqrt{\frac{1}{n}\left(I^{-1}\right)_{v v}}
$$

$$
t=\frac{\hat{v}-v_{0}}{e e(\hat{v})}
$$

el estadístico de prueba para el test correspondiente que verifica la significancia del parámetro estimado.

Finalmente, este conjunto de resultados se acompañan con la prueba de hipótesis de Kolmogorov (Ross, 2012), así se complementa la bondad de ajuste de la DHG sobre la muestra aleatoria y se encuentra evidencia hacia la distribución poblacional.

En la siguiente sección se presentan las estimaciones y la discusión de los resultados para la serie de rendimientos de los precios del petróleo WTI, 
Brent y MME en el periodo 2010-2015. El objetivo es caracterizar el comportamiento de los rendimientos mediante los parámetros de la DHG para los subperiodos de precios bajos y precios altos.

\section{Estimaciones y resultados}

Los rendimientos diarios $x_{i t}$ de los precios del petróleo WTI, Brent y MME se han calculado bajo la siguiente expresión:

$$
x_{i t}=\ln \left(p_{i t}\right)-\ln \left(p_{i t-1}\right),
$$

donde $i=1,2,3$ son los precios del petróleo y $t$ es la variable tiempo que abarca los días durante los años 2010-2015. Entonces

$p_{i t}=$ precio diario observado del tipo de petróleo $i$ en el tiempo $t$,

$x_{i t}=$ rendimiento diario del precio del tipo de petróleo $i$ en el tiempo $t$.

Para comenzar con el análisis se presentan los estadísticos descriptivos en el cuadro 1. Se puede observar que los rendimientos de WTI presentan una volatilidad superior, en tanto que MME muestra el rendimiento medio más bajo.

\section{Cuadro 1}

Estadísticos descriptivos de los rendimentos

\begin{tabular}{|lcccc|}
\hline Variable & Media & $\begin{array}{c}\text { Desviación } \\
\text { estándar }\end{array}$ & Mínimo & Máximo \\
\hline WTI & $-0.067 \%$ & $2.153 \%$ & $-11.603 \%$ & $11.621 \%$ \\
BRENT & $-0.059 \%$ & $1.946 \%$ & $-9.519 \%$ & $10.416 \%$ \\
MNE & $0.073 \%$ & $1.824 \%$ & $-13.861 \%$ & $11.384 \%$ \\
\hline
\end{tabular}

Fuente: elaboración propia con datos de Pemex.

En el cuadro 2 las correlaciones entre los diferentes rendimientos es positiva y relativamente elevada, lo que justifica su impacto directo sobre la mezcla mexicana de exportación. 
Cuadro 2

Matriz de correlación para los rendimientos

\begin{tabular}{|l|lll|}
\hline & WTI & BRENT & MME \\
\hline WTI & 1 & 0.7733 & 0.7306 \\
BRENT & 0.7733 & 1 & 0.7513 \\
MME & 0.7306 & 0.7513 & 1 \\
\hline
\end{tabular}

Fuente: elaboración propia con datos de Pemex.

En el cuadro 3 se presenta la prueba aumentada de Dickey-Fuller para los rendimientos de los precios del petróleo, se puede apreciar que para cada subperiodo las series de tiempo no presentan raíz unitaria, pues se rechaza la hipótesis nula. En otras palabras, las series de datos son estacionarias.

Cuadro 3

Prueba de raíz unitaria para los rendimientos

\begin{tabular}{|lllll|}
\hline \multicolumn{1}{|c}{$\begin{array}{c}\text { Tipos de } \\
\text { petróleo }\end{array}$} & \multicolumn{2}{c}{ 2010-2013 } & \multicolumn{2}{c|}{ 2014-2015 } \\
\hline WTI & -10.0702 & 0.0000 & -10.8362 & 0.0000 \\
BRENT & -11.8232 & 0.0001 & -8.1185 & 0.0000 \\
MME & -10.5498 & 0.0000 & -9.2059 & 0.0000 \\
\hline
\end{tabular}

Fuente: elaboración propia con datos de Pemex.

Las estimaciones de los parámetros para los subperiodos 2010-2013 y 2014-2015 se muestran en el cuadro 4. Entre paréntesis se reporta el estadístico $t$ correspondiente para verificar si el parámetro es estadísticamente significativo. De hecho, se puede observar que los coeficientes son significativos al menos al $90 \%$ de nivel de confianza.

En el cuadro 4 también se reportan los valores $p$ asociados a la prueba de Kolmogorov, tanto para la distribución normal como para la función de densidad hiperbólica generalizada. Nótese que existe evidencia para afirmar que los rendimientos de los precios del petróleo siguen cualitativamente la misma distribución en ambos periodos, salvo que bajo diferentes parámetros. Más aún, la distribución normal se encuentra muy lejos de ajustar a la muestra de datos. 


\section{Cuadro 4}

Estimación de los parámetros de la DHG y bondad de ajuste

\begin{tabular}{|cccccccccc|}
\hline & \multicolumn{7}{c|}{ 2010-2013 } \\
\hline $\begin{array}{c}\text { Tipos } \\
\text { de } \\
\text { petróleo }\end{array}$ & $u$ & $\delta$ & $a$ & $\beta$ & $\lambda$ & $\begin{array}{c}\text { Log } \\
\text { verosimilitud }\end{array}$ & DHG & Normal \\
\cline { 2 - 9 } ajuste (valor $p$ )
\end{tabular}

Fuente: elaboración propia con datos de Pemex.

El cambio en los parámetros de la distribución para ambos subperiodos se puede resumir como sigue:

a) El parámetro $\beta$ disminuye en magnitud y tiende a ser mayor que cero, lo que indica un sesgo en la distribución de rendimientos. Además, cambia el nivel de la volatilidad, ya que este parámetro influye directamente en la varianza. Asimismo, este movimiento junto con la caída de $\alpha$ refleja que la moda de la variable rendimiento es negativa para el tramo 2014-2015. 
b) $\mu$ mide la ubicación del valor esperado, así que claramente el valor medio poblacional de los rendimientos es menor a cero en el periodo 2014-2015, pero a un nivel de volatilidad diferente al que se observaba en 2010-2013, pues el valor $\beta$ ha cambiado de nivel.

c) $\delta$ es un parámetro que no cambió sustancialmente entre los periodos, así que la concentración de los rendimientos alrededor de la moda es equivalente tanto en precios altos como en precios bajos.

d) $\lambda$ señala que el nivel de dispersión alrededor del valor esperado no ha cambiado hacia una cola particular de la distribución. Es decir, que a pesar de los rendimientos negativos y la volatilidad del periodo 20142015, los rendimientos poblacionales se ubicarán alrededor de la moda en el corto plazo, sólo a que a un nuevo nivel de media-varianza.

En general, se puede afirmar que el seguimiento a los parámetros de la distribución que siguen los rendimientos del precio del petróleo, permite inferir más allá de la muestra el comportamiento en el corto plazo. Esto es relevante, pues se puede analizar el conjunto de rendimientos y evitar sobreestimaciones o subestimaciones de la volatilidad, que pudieran atribuirse a la percepción diaria de los precios del petróleo.

\section{Conclusiones}

Los precios del petróleo han variado drásticamente en los últimos años, de ahí que analizar la distribución de probabilidad de los rendimientos sea un ejercicio necesario. En este trabajo se ha encontrado evidencia de que la distribución de probabilidad hiperbólica generalizada se ajusta razonablemente a los casos WTI, Brent y MME. Concretamente, se tiene evidencia mediante la prueba de Kolmogorov y bajo una prueba estadística asintótica para valorar la significancia de cada parámetro estimado.

Dado que se cuenta con una distribución de probabilidad adecuada para los rendimientos de los precios del petróleo, tanto con precios altos como con precios bajos, entonces es posible caracterizar el comportamiento de los rendimientos mediante el cambio que experimentan sus parámetros.

En particular, en este documento se puede señalar que bajo los parámetros calculados, el comportamiento de los rendimientos presenta la misma estructura cualitativa que cuando se tenían precios altos, salvo que la 
dispersión, el sesgo, la curtosis y la moda han cambiado de nivel. Esto da cuenta de que los precios se han situado en un nuevo contexto (parámetros diferentes en DGH), lo que se traduce poblacionalmente en que los rendimientos se rigen bajo otros parámetros. Este escenario poblacional nuevo, se tiene una media y moda más pequeñas, así que parecería que los tiempos de precios de barril de petróleo mayores a \$100 dólares no regresarán, salvo un choque fuerte que cambie los parámetros drásticamente.

Estos resultados sugieren un nuevo escenario de precios de petróleo (bajos) hacia el largo plazo. 


\section{Referencias}

Barndorff-Nielsen, O. E. (1977). "Exponentially decreasing distributions for the logarithm of the particle size". Proceedings of the Royal Society, Series A, Mathematical and Physical Sciences, 353(1674), pp. 401-419.

Barndorff-Nielsen, O. E.; T.,Mikosch, y S. I. Resnick, (Eds.) (2012). Lévy processes: theory and applications. Springer Science \& Business Media, pp. 47-93.

Brown, Jonathan (1993). Oil and revolution in Mexico, University of California Press, United States, pp. 33-68.

Cárdenas-Gracia, Jaime (2009). En defensa del petróleo, Universidad Nacional Autónoma de México, México, pp. 120-145.

Centro de Estudios de las Finanzas Públicas (2009). "Precios del petróleo mexicano: Expectativas y volatilidad", disponible en: http://www.cefp.gob.mx.

Greene, W. H. (2012). Econometric Analysis. Boston: Prentice Hall, pp. 150-190.

Hamilton, J. D. (1994). Time Series Analysis. Princeton, N. J.: Princeton University Press, United States, pp. 88-130.

$\mathrm{Hu}$, W. (2005). “Calibration of Multivariate Generalized Hyperbolic Distributions using the EM Algorithm, with Applications in Risk Management, Portfolio Optimization and Portfolio Credit Risk". Electronic Theses, Treatises and Dissertations, Paper 3694, pp. 40-78.

Instituto Mexicano del Petróleo (2011). “Tipos de petróleo”, disponible en: http:// www.imp.mx.

Paolella, M. S. (2007). Intermediate Probability. West Sussex, England: John Wiley \& Sons, pp. 28-52.

Protassov, R. S. (2004). “EM-based maximum likelihood parameter estimation for multivariate generalized hyperbolic distributions with $\lambda$ fixed". Statistics and Computing, 14(1), pp. 67-77.

Ross, S. M. (2012). Simulation. Estados Unidos: Academic Press, pp. 173-220.

Secretaría de Energía (2012). “Prospectiva de Petróleo y Petrolíferos 2013-2027" disponible en: http://sener.gob.mx.

Tsay, R. S. (2010). Analysis of financial time series. Cambridge, Mass.: Wiley, pp. 230-280. 